\title{
EFECTO DE DIFERENTES TRATAMIENTOS DE DESINFECCIÓN DEL SUELO SOBRE LAS PROPIEDADES EDÁFICAS
}

\section{EFFECT OF DIFFERENT SOIL DISINFECTION TREATMENTS ON EDAPHIC PROPERTIES}

\author{
Marisol Cuellas ${ }^{1 *}$, Paula Amoia ${ }^{1}$, y Pablo Delmazzo ${ }^{1}$ \\ ${ }^{1}$ Agencia de Extensión La Plata, Estación Experimental del Área Metropolitana de Buenos Aires, \\ Instituto Nacional de Tecnología Agropecuaria (INTA), Ruta 36 Km 44,5 (1900 La Plata), Argentina. \\ * Autor para correspondencia E-mail: cuellas.marisol@inta.gob.ar
}

\section{RESUMEN}

En la región del Gran La Plata se produce bajo cubierta alrededor de 4.600 ha de cultivos agrícolas, sobre suelos Typic Hapludert y Vertic Argiudol, caracterizados por un drenaje deficiente, que junto a la calidad del agua de riego y al manejo intensivo, conducen a degradaciones físicas, químicas y biológicas, que se vinculan con una elevada carga de patógenos (enfermedades y nematodos). Por lo tanto, uno de los desafíos más importantes es encontrar alternativas de desinfección de suelo que se adapten al sistema productivo, que no contaminen ni degraden los recursos, y que promuevan una mejora de los suelos. En el presente trabajo se evaluó el efecto de diferentes tratamientos de desinfección en las propiedades edáficas $(\mathrm{pH}, \mathrm{CE}, \mathrm{MO}, \mathrm{RAS}, \mathrm{Ca}, \mathrm{Mg}$ ) y en el rendimiento de un cultivo. Los tratamientos evaluados fueron: solarización, biosolarización con cama de pollo (50 $\left.t \mathrm{ha}^{-1}\right)$, biosolarización con cama de champiñón $(100 \mathrm{t}$ ha-1), biosolarización con repollo $(80 \mathrm{t}$ ha1), desinfección química y un testigo, en dos temporadas (2014 y 2015) de un cultivo de tomate. Se observó que la biosolarización con cama de pollo y de champiñón aumentó los niveles de salinidad y las concentraciones de $\mathrm{Ca}^{2+} \mathrm{y} \mathrm{Mg}^{2+}$, sin una mejora significativa en los niveles de MO. La biosolarización con repollo no incidió en las variables del suelo. Los tratamientos de solarización y desinfección química se comportaron en forma similar al testigo. Respecto al rendimiento del cultivo la desinfección química presentó los mayores valores. El resto de los tratamientos no se diferenciaron significativamente $(P<0,05)$ entre sí.

Palabras clave: solarización, biosolarización, tomate, cama de pollo, cama de champiñón, repollo, salinización, enmienda orgánica, producción bajo cubierta.

\section{ABSTRACT}

In the Gran La Plata region, around 4,600 ha of agricultural crops are grown undercover on Typic Hapludert and Vertic Argiudol soils, characterized by poor drainage because of their high content of expandable clay. The characteristics of the soils, together with the quality of irrigation water and intensive management lead to physical, chemical and biological degradation associated with the presence of abundant pathogens (diseases and nematodes). Therefore, it is one of the most important challenges to find alternatives for soil disinfection that can be adapted to this type of production system, prevent contamination or degradation of resources, and promote soil improvement. This study evaluated the effect of different soil disinfection treatments on the soil properties ( $\mathrm{pH}, \mathrm{EC}, \mathrm{MO}$, RAS, Ca, Mg) and crop yield. The treatments tested were: solarization, biosolarization with chicken manure $\left(50 \mathrm{t} \mathrm{ha}^{-1}\right)$, biosolarization with mushroom residues $\left(100 \mathrm{t} \mathrm{ha}^{-1}\right)$, biosolarization with cabbage 
residues $\left(80 \mathrm{tha}^{-1}\right)$, chemical disinfection and a control. The experiment was conducted during two seasons (2014 and 2015) of a tomato crop. It was observed that biosolarization with chicken manure and mushroom residues increased salinity levels and concentrations of $\mathrm{Ca}^{2+}$ and $\mathrm{Mg}^{2+}$, without a significant improvement in OM levels. Biosolarization with cabbage residues did not affect the soil variables. Solarization and chemical disinfection treatments behaved similarly to the control. Regarding crop yield, chemical disinfection presented the highest values. No significant differences $(P<0.05)$ were found between the rest of the treatments.

Key words: solarization, biosolarization, chicken manure, mushroom, cabbage, tomato, salinization, organic amendment, production under cover.

\section{INTRODUCCIÓN}

En el cinturón hortícola del Gran La Plata, Buenos Aires, Argentina, se concentran alrededor de 4.600 ha en producción bajo cubierta (Miranda, 2017), que abastecen de alimento fresco a los principales centros de consumo, conformando una de las regiones de producción hortícola periurbana más importante del país. Si bien la cercanía a las grandes ciudades constituye una ventaja competitiva para esta región, la forma de producción intensiva (como excesos de fertilización, uso de plaguicidas sin control, etc.) para satisfacer la demanda creciente (tanto en calidad como en cantidad), producen diversas problemáticas en el sistema productivo que se vinculan principalmente a los procesos tecnológicos, y que con el tiempo provocan la degradación de los recursos naturales (Polack, 2013).

Los suelos donde se desarrolla la actividad hortícola, se clasifican como Typic Hapludert y Vertic Argiudol; en su condición natural presentan elevada fertilidad química y materia orgánica $(4,5-5 \%)$, están libres de sales y sodio $\left(\mathrm{CE}<1 \mathrm{dS} \mathrm{m}^{-1}\right)$, relación de adsorción de sodio (RAS) < 1, pH ligeramente ácido $(\mathrm{pH} 5,5), \mathrm{y}$ contenido de $\mathrm{P}$ bajo $\left(<10 \mathrm{mg} \mathrm{kg}^{-1}\right)$, su principal limitante es la permeabilidad baja a moderada, debido al elevado contenido de arcillas (Hurtado et al., 2006). Estas características sumadas al riego con agua de baja calidad (bicarbonatada sódica) (Alconada y Zembo, 2000) y al manejo productivo (exceso de fertilización y aplicación de enmiendas orgánicas e inorgánicas sin control), son las causas principales que los degradan (Alconada, 2004; Poncetta et al., 2006). Se han reportado valores promedios de CE $4 \mathrm{dS}$ $\mathrm{m}^{-1}$, sodificación (PSI 18\%), pérdida de materia orgánica (promedio 3,4\%) e hiperfertilización (concentraciones de $\mathrm{P}$ hasta $535 \mathrm{mg} \mathrm{kg}^{-1}$ ), con síntomas de deficiencias inducidas en los cultivos por el desbalance nutricional (Cuellas, 2017). Así, con frecuencia los cultivos de fruto, como tomate (Solanum lycopersicum) y pimiento (Capsicum annuum), presentan deficiencias de
$\mathrm{Ca}^{2+}$ denominada blossom end rot (BER) (necrosis en el sector apical de los frutos). La misma puede tener distintos orígenes, más que corresponderse específicamente a un bajo contenido de $\mathrm{Ca}^{+2}$ edáfico, como excesos iónicos $\left(\mathrm{K}^{+}, \mathrm{Na}^{+}, \mathrm{Mg}^{+2}, \mathrm{y}\right.$ $\left.\mathrm{NH}_{4}^{+}, \mathrm{Mn}^{+2}\right)$, estrés por agua, alta salinidad y alta humedad relativa (Ho and White, 2005; del Amor and Marcelis, 2006). Esta problemática no solo se presenta en la región de estudio, sino también en otros sitios del mundo con situaciones productivas similares. Al respecto, Rubio et al. (2009) observaron una reducción del rendimiento y de frutos comerciales, principalmente por un aumento del número de frutos con blossom end rot (BER) en condiciones de salinidad. Se destacan entonces en la región las degradaciones físicas, químicas y biológicas de los suelos (Alconada et al., 2011), que favorecen la mayor incidencia de enfermedades y plagas tanto en los cultivos como en el suelo (Altieri and Nicholls, 2003).

Por lo tanto, uno de los desafíos productivos más importantes de esta región y de otras áreas hortícolas que afrontan problemáticas similares, es encontrar alternativas de desinfección de suelo (control de enfermedades y nematodos) que sean económicas y socialmente viables, de bajo impacto ambiental (Bello et al., 2000; Bello et al., 2008), y que se adecuen a cada sistema productivo, promoviendo además una mejora en las condiciones edáficas.

Dentro de las alternativas de desinfección física y biológica, las más ensayadas son la solarización, cuyo control se basa en el aumento de la temperatura del suelo por medio de la radiación solar, y la biofumigación, que consiste en la utilización de gases fumigantes que resultan de la degradación de la materia orgánica y otros residuos agrarios biológicos (Bello et al., 2008). La combinación de ambas técnicas es la biosolarización, muy utilizada debido a que incrementa la eficacia de cada una (las altas temperaturas acentúan la liberación de compuestos volátiles de los residuos orgánicos) (Ploeg et al., 2001). Existen diferentes posibilidades de subproductos y de materiales orgánicos que se pueden incorporar al suelo como biofumigantes, que no sólo realizan el control de patógenos 
sino que también pueden mejorar la estructura y la fertilidad de los mismos (Bonanomi et al., 2007). No obstante, antes de su incorporación se deben considerar sus características físicas, químicas y biológicas. Sin embargo, en la gran mayoría de los casos, se aplican sin análisis previo, produciendo con el tiempo efectos adversos (Pérez et al., 2008), como contaminación y degradación de los recursos naturales y daño en los cultivos (Rabuffetti et al., 2010). Por lo tanto la aplicación sucesiva de ciertos productos, plantea la necesidad de abordar el estudio teniendo en consideración aspectos fundamentales, como su efecto sobre el suelo y su impacto en el medio ambiente (Fernández et al., 2014). Una de las limitantes más importantes que se observa son las grandes cantidades requeridas para el control satisfactorio, que en general resultan fitotóxicas o no son rentables (Oka, 2010). Se recomiendan dosis de productos orgánicos (con una relación C/N 8-20) de $50 \mathrm{t} \mathrm{ha}^{-1}$, y cuando los problemas de nematodos $\mathrm{u}$ hongos son muy graves dosis de 100 t ha ${ }^{-1}$ (Bello et al., 2000).

El agregado de materiales orgánicos influyen en la dinámica y en el contenido de nutrientes del suelo (Di Gioia, 2017). Diferentes ensayos en los cuales se han utilizado enmiendas orgánicas (estiércol de animal) para biofumigar reportan aumento de salinidad, que afectan las propiedades edáficas y el desarrollo de los cultivos (Dikinya and Mufwanzala, 2010; Borahona et al., 2015). En condiciones de salinidad, la planta sufre desbalance nutricional, déficit hídrico y toxicidad por iones específicos, que afectan directamente su rendimiento (Grattan and Grive, 1999; Porta et al., 2003). No obstante, es importante considerar que cada cultivo presenta una CE (conductividad eléctrica) óptima para su desarrollo. Así por ejemplo, en el cultivo de tomate se observa que a partir de concentraciones salinas de 2-2,5 dS m ${ }^{-1}$ el rendimiento se verá afectado (Nuez et al., 2003), con una reducción del $6 \%$ por cada unidad de $\mathrm{CE}$ que se incrementa por encima de estos valores (Maggio et al., 2007).

El $\mathrm{pH}$ del suelo también se modifica. Al incorporar productos orgánicos con elevada presencia de $\mathrm{N}$, como la cama de pollo (estiércol de gallina + cáscara de arroz) y gallinaza (estiércol de gallina puro), se produce inicialmente un descenso del pH (del Pino et al., 2007; Oka, 2010), ocasionado por la liberación de $\mathrm{H}^{+}$durante el proceso de nitrificación (Mengel and Kirkby, 2000), que se intensifica en suelos alcalinos (Oka, 2010). Sin embargo, de acuerdo al tipo de material que se incorpora el $\mathrm{pH}$ también puede aumentar por la presencia de cationes básicos que se liberan durante su descomposición (Dikinya and Mufwantala, 2010; Simmons et al., 2016). Es muy importante que el suelo tenga un $\mathrm{pH}$ que permita el desarrollo óptimo de los cultivos; para el tomate el rango se encuentra entre 5,5-6,5 (Nuez et al., 2003), valores por encima de este nivel afectan la disponibilidad de ciertos nutrientes y en consecuencia el rendimiento del cultivo (Porta et al., 2003).

Respecto a la modificación de la materia orgánica (MO) del suelo, se espera que la incorporación de enmiendas orgánicas tenga un efecto positivo promoviendo su aumento y en consecuencia mejorando la estructura del suelo y permeabilidad (Pung, 2003). No obstante, hay que considerar que la generación de materia orgánica depende del tipo de material incorporado, ya que la formación de humus se produce principalmente a partir de materiales de origen vegetal (Labrador Moreno, 1996). Al respecto, en un estudio realizado por Mitidieri et al. (2009) no se encontraron cambios significativos en las concentraciones de $\mathrm{MO}$ al comparar diferentes tratamientos de biosolarización.

Por lo tanto es muy importante no sólo centrar las investigaciones en el control fumigante de las enmiendas incorporadas, sino que también se debe analizar que sucede en el suelo una vez que se incorporan. El presente trabajo tuvo como objetivo comparar el efecto de la aplicación de diferentes tratamientos de desinfección de suelo en las propiedades edáficas, en el rendimiento del cultivo y en la incidencia de BER en los frutos.

\section{MATERIALES Y MÉTODOS}

Ubicación y características productivas. El ensayo se realizó en la Estación Experimental de Gorina del Ministerio de Agroindustria de la Provincia de Buenos Aires, ubicada en el Gran

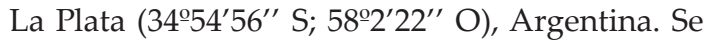
usaron dos invernáculos parabólicos de metal (240 $\mathrm{m}^{2}$ ), durante dos ciclos productivos (2014 y 2015) de un cultivo de tomate (Solanum lycopersicum) var. Elpida. El manejo implementado fue de tipo tradicional, conforme a las recomendaciones frecuentes para la región: densidad de plantación 18.000 plantas ha-1, riego por goteo $(20 \mathrm{~cm}$ entre goteros) con dos mangueras de riego por lomo.

Caracterización inicial del sitio. Las propiedades analíticas se realizaron por procedimientos estándares (Page et al., 1982), y el fósforo (P) asimilable por el método de Bray Kurtz 1, clasificando el suelo por Soil Taxonomy (Soil Survey Staff, 2006).

Tratamientos. T1 Testigo; T2 Solarización; T3 Biosolarización con cama de pollo (CP) (mezcla de estiércol de pollo $(40 \% \mathrm{v} / \mathrm{v})+$ cascarilla de 
$\operatorname{arroz}(60 \% \mathrm{v} / \mathrm{v})$, dosis 50 tha $\left.^{-1}\right)$; T4 Biosolarización con cama de champiñón $(\mathrm{CCH})$ (mezcla cama de pollo + cama de stud (estiércol de caballo (30-40\% v/v) + viruta de salicáceas $(60-70 \%$ v/v $))+$ urea + melaza, dosis $100 \mathrm{t} \mathrm{ha}^{-1}$ ); T5 Biosolarización con repollo (Brassica oleracea) var. capitata (el repollo se trituró y se aplicó picado sobre el lomo) dosis $80 \mathrm{t} \mathrm{ha}^{-1}$; y T6 Desinfectante químico (1,3 Dicloropropeno + cloropicrina, dosis $50 \mathrm{gr}$ $\left.\mathrm{m}^{-2}\right)$. Se destaca que el sitio donde se instaló el ensayo presentaba una alta carga de nematodos (valores promedios ( $\mathrm{N}^{\circ}$ individuos/100 cc suelo): 12,3 Nacobus aberrans, 9,8 Tylenchorhynchus y 5,4 Pratylenchus), por tal motivo las dosis de los tratamientos se establecieron de acuerdo a lo recomendado en estas situaciones para el control de los mismos (Bello et al., 2000).

El suelo se preparó con los manejos habituales. Secuencia de labores: cincelado, rastra rotativa y construcción de lomos (tamaño 0,80 m de ancho y $5 \mathrm{~m}$ de largo). Una vez realizada estas labores, se procedió a la instalación de los diferentes tratamientos. En el T2 (solarización) se regó y tapó con un polietileno cristal de 50 micrones, y en los tratamientos donde se aplicó la biosolarización (T3, T4 y T5) se realizaron las mismas labores a excepción que antes del riego se incorporaron las enmiendas (el 21/11/13 para el ciclo 2014 y el 5/12/14 para el ciclo 2015). El período que los tratamientos permanecieron tapados fue de 45 días. Una vez transcurrido ese tiempo se destapó y a los 5 días se procedió al trasplante. Para el T6, una vez preparados los lomos y tapados, se incorporó el 1,3 Dicloropropeno + cloropicrina. Este tratamiento permaneció tapado durante 15 días y se dejó 10 días de ventilación antes de plantar. La plantación se realizó en ambos ciclos durante el mes de enero.

Diseño experimental y análisis estadístico. El diseño experimental fue en bloques al azar con 4 repeticiones. Se analizaron los resultados por ANVA, con prueba de $\mathrm{F}$ test unilateral $(\mathrm{P}<0,05$ y 0,01$)$, comparación de tratamientos por test de Duncan, mediante el programa InfoStat (InfoStat, 2004).

Muestreo y análisis de suelo. Se tomaron muestras compuestas (formadas por cuatro submuestras), durante los dos ciclos productivos un total de 6 muestreos (72 muestras), profundidad de muestreo de $0-15 \mathrm{~cm}$.

Muestreo inicial previo a los tratamientos. F1 (Diciembre 2013) y F4 (Diciembre 2014). Muestreo compuesto en cada uno de los sectores en los que se instalaron los tratamientos y el testigo.

Muestreos durante el desarrollo del cultivo. Luego de instalados los tratamientos, se efectuaron 2 muestreos: durante el desarrollo del cultivo, y al final del ciclo, durante los dos ciclos del cultivo estudiado, en las siguientes fechas: F2 (Abril 2014), F3 (Agosto 2014), F5 (Abril 2015), F6 (Agosto 2015). Las muestras correspondientes al desarrollo del cultivo y al final del ciclo se tomaron por repetición en los tratamientos. En los muestreos realizados al inicio (F1 y F4) del ciclo del cultivo, se tomó una muestra compuesta de cada tratamiento.

Se analizaron las siguientes variables por estándares de evaluación (Page et al., 1982): pH en pasta, conductividad eléctrica (CE) en el extracto de suelo a saturación, capacidad de intercambio catiónico (CIC) por extracción con acetato de amonio $1 \mathrm{~N} \mathrm{pH}$ 7. Cationes solubles: extracción y posterior evaluación con la metodología indicada para cada catión: fotometría en llama para sodio $\left(\mathrm{Na}^{+}\right)$y colorimetría para calcio $\left(\mathrm{Ca}^{+2}\right)$ y magnesio $\left(\mathrm{Mg}^{+2}\right)$; y materia orgánica $(\mathrm{MO})$ por Walkley y Black.

Muestreo y análisis de las enmiendas. Se tomaron muestras compuestas, en los dos ciclos productivos, de la cama de pollo y de la cama de champiñón. La caracterización se realizó utilizando la metodología de análisis de sustrato del laboratorio del Instituto de Floricultura, INTA Castelar.

Análisis en el cultivo. En cada ciclo productivo se analizó el rendimiento de cada tratamiento y se evaluó el número de frutos con blossom end rot (BER).

\section{RESULTADOS Y DISCUSIÓN}

Suelo inicial (F1). El suelo descripto en el sitio de trabajo (dentro del invernáculo) se clasificó como Typic Hapludert. Presentó un horizonte A $(15 \mathrm{~cm}$ de espesor), inmediatamente debajo se observó un Btss con elevado contenido de arcillas, con alta proporción de tipo expansiva que resultaron suficientes como para generar slickensides (superficie brillante con estrías causada por el deslizamiento entre masas de suelo). Se constató en superficie una elevada salinidad, porcentaje de sodio intercambiable, alcalinidad, y P asimilable que se correspondieron con valores de CE 5,3 dS m-1 ${ }^{-1}$ PSI 18,9, pH 6,9, y P $305 \mathrm{mg} \mathrm{kg}^{-1}$, que revela un proceso manifiesto de degradación, coincidente con lo indicado para la región en estos esquemas productivos por el uso excesivo de agroquímicos, abonos, manejo del suelo y agua de riego (Alconada y Zembo, 2000; Giuffré et al., 2004; Alconada et al., 2011; Cuellas, 2017). Este suelo, dista marcadamente de la condición natural del suelo descripta por Hurtado et al. (2006), que como se comentó presenta como limitantes un drenaje imperfecto y bajo contenido 
de $\mathrm{P}\left(10 \mathrm{mg} \mathrm{kg}^{-1}\right)$, sin sales ni álcali, bajo $\mathrm{pH}$, y una elevada fertilidad química.

Análisis de las enmiendas. Los resultados del análisis químico de las enmiendas $\mathrm{CP}$ (cama de pollo) y $\mathrm{CCH}$ (cama de champiñón) para el 2014 y 2015 se presentan en la Tabla 1. Si bien el origen fue el mismo en ambos ciclos, se observa variabilidad en su composición. Lo cual reafirma la importancia de realizar un análisis en cada situación y no basarse en análisis previos y en la bibliografía para tomar decisiones (Alconada, 2004). Es también importante tener en consideración que debido a esta variabilidad existen grandes dificultades en predecir su efecto en cada situación (del Pino et al., 2007).

Se observó en ambas camas un pH elevado y una elevada salinidad, destacándose la $\mathrm{CP}$ en el 2015 con valores más extremos ( $\mathrm{pH}$ 9,3; CE $8,8 \mathrm{dS} \mathrm{m}^{-1}$ ). Estos valores fueron similares a los reportados por Alconada (2004) en la región de estudio y se vinculan con la salinización y alcalinización de los suelos (Alconada et al., 2011; Cuellas, 2017). En general la CCH presentó una mayor riqueza en los cationes principalmente de $\mathrm{Ca}^{2+} \mathrm{y} \mathrm{K}^{+}$y la $\mathrm{CP}$ mayor concentración de $\mathrm{K}^{+}$en el 2015 (Tabla 1). Por lo tanto, en muchos casos la aplicación de estas enmiendas puede superar las necesidades nutricionales de los cultivos, aumentando su acumulación en los horizontes superficiales, generando así desbalances nutritivos, y aumentando también el riesgo de contaminación de las aguas superficiales (Rabuffetti et al., 2010).

Reacción del suelo $(\mathrm{pH})$. Los valores de $\mathrm{pH}$ para los tratamientos en los dos ciclos de evaluación (2014 y 2015) se presentan en la Tabla 2. Se observa al inicio del ensayo (antes de la incorporación de los tratamientos) que el $\mathrm{pH}$ del suelo se encontraba cercano a la neutralidad (F1, 6,9), valor que resultó más elevado que los reportados por Hurtado et al. (2006) en su condición natural $(\mathrm{pH} 5,5)$. Hacia F2 se produjo un aumento en todos los tratamientos (rango 7,2-7,7) sin diferenciarse significativamente entre sí. En F3 los valores fueron similares a F2, sin embargo se presentó diferencia significativa $(\mathrm{P}<0,05)$ entre

Tabla 1. Análisis químico de las enmiendas orgánicas. Años 2014 y 2015.

Table 1. Chemical analysis of organic amendments. Seasons 2014 and 2015.

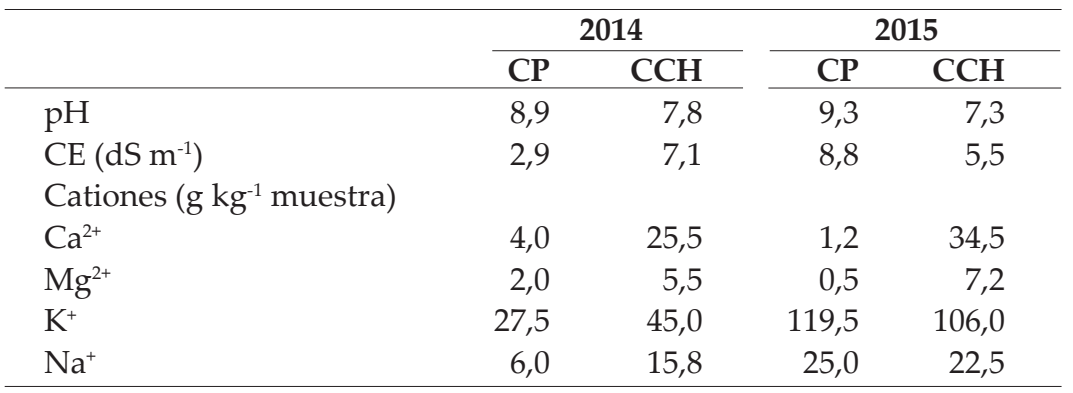

CP: Cama de pollo; $\mathrm{CCH}$ : Cama de champiñón.

Tabla 2. Valores de $\mathrm{pH}$ para cada tratamiento y fecha de evaluación.

Table 2. $\mathrm{pH}$ values for each treatment and season evaluated.

\begin{tabular}{|c|c|c|c|c|c|c|c|}
\hline & T1 & T2 & T3 & T4 & T5 & T6 & $\mathrm{CV}$ \\
\hline & \multicolumn{7}{|c|}{2014} \\
\hline F1 & 6,90 & 6,90 & 6,90 & 6,90 & 6,90 & 6,90 & \\
\hline F2 & 7,69 Аа & 7,61 Aa & 7,57 Aa & 7,21 Аа & 7,26 Аа & 7,42 Aa & 3,43 \\
\hline F3 & 7,82 Ba & 7,69 ABa & $7,49 \mathrm{ABa}$ & 7,17 Аа & $7,28 \mathrm{ABa}$ & 7,88 Ba & 3,53 \\
\hline \multirow[t]{2}{*}{$\mathrm{CV}$} & 1,55 & 5,81 & 2,65 & 3,71 & 3,55 & 2,74 & \\
\hline & \multicolumn{7}{|c|}{2015} \\
\hline F4 & 7,84 & 7,51 & 8,00 & 7,63 & 7,66 & 7,85 & \\
\hline F5 & 7,91 Ca & 7,96 Ca & $7,45 \mathrm{Aa}$ & 7,58 ABa & 7,61 ABa & 7,83 BCa & 2,34 \\
\hline F6 & 7,92 ABa & $8,09 \mathrm{Ba}$ & 7,76 ABa & 7,29 Аа & $7,86 \mathrm{ABa}$ & $8,15 \mathrm{Ba}$ & 4,16 \\
\hline CV & 5,48 & 2,98 & 3,72 & 2,85 & 5,11 & 2,51 & \\
\hline
\end{tabular}

Letras mayúsculas iguales entre tratamientos no difieren entre sí.

Letras iguales minúsculas entre fechas no difieren entre sí. 
los sitios evaluados, así el T1 (pH 7,8) y T6 (pH $7,9)$ se diferenciaron del T4 $(\mathrm{CCH})(\mathrm{pH} 7,2)$.

El ciclo 2015 se inició con valores más elevados (F4, pH promedio 7,7) respecto del 2014 (Tabla 2) y se observa en las siguientes fechas de evaluación (F5 y F6), que al igual que el ciclo anterior, los tratamientos en los cuales se incorporó enmienda orgánica $(\mathrm{CP}$ y $\mathrm{CCH})$ presentaron los valores más bajos, esto probablemente se debió al proceso de nitrificación y de mineralización del carbono orgánico, generando amonio, poder reductor y ácidos orgánicos, como efecto de la incorporación de camas con elevado contenido de $\mathrm{N}$ y baja relación C/N (Mengel and Kirkby, 2000; Oka, 2010). Así en F5 el T1 y T2 con valores próximos a 8,1 se diferenciaron significativamente $(P \leq 0,05)$ del T3 (pH 7,4) y T4 (pH 7,6) y sólo el T3 del T6 $(\mathrm{pH} 8,3)$. Hacia F6 se produjo un aumento de $\mathrm{pH}$ sin diferencia significativa entre fechas (rango entre 7,3 y 8,15), y el $\mathrm{T} 4$ con menor valor $(\mathrm{pH}$ $7,3)$ se diferenció $(\mathrm{P} \leq 0,05)$ del T2 y T6 (ambos tratamientos $\mathrm{pH}$ próximos a 8,1). Del Pino et al. (2007) también reportan la tendencia decreciente de $\mathrm{pH}$ en los suelos con el agregado de cama de pollo. Se destaca además que el proceso de nitrificación se produce más rápidamente en suelos alcalinos como los que se observaron en F4 (Oka, 2010). Respecto al T5 (biosolarización con repollo) tuvo un comportamiento intermedio sin diferenciarse significativamente (excepto en F5) del resto de los sitios. Similares resultados fueron reportados por Mitidieri et al. (2009) al comparar la biofumigación con repollo vs solarización y aplicación de estiércol.

Se observó en todos los tratamientos que el suelo va sufriendo un proceso de alcalinización; los valores del presente estudio fueron similares a los reportados por Cuellas (2017) para condiciones de manejo tradicional en la región de estudio ( $\mathrm{pH}$ promedio 7,5). El aumento de $\mathrm{pH}$ se pudo deber principalmente a las características del agua de riego $(\mathrm{pH} 7,7)$ (Alconada y Zembo, 2002; Cuellas, 2015) y en los tratamientos de biosolarización al $\mathrm{pH}$ elevado de las camas con elevado contenido de cationes básicos (Dikinya and Mufwantala, 2010; Simmons et al., 2016) (Tabla 1). Se destaca que el cultivo de tomate tiene un óptimo de crecimiento en un rango de pH entre 5,5-6,5 (Nuez et al., 2003), por lo tanto los valores alcanzados en el ensayo pudieron afectar la disponibilidad de ciertos nutrientes y el desarrollo del cultivo (Porta et al., 2003).

Salinidad (CE). Los valores de CE para todos los tratamientos y fechas de evaluación se presentan en la Tabla 3. Se observa que durante el ciclo 2014 (F2 y F3) no hubo diferencia significativa entre tratamientos, sin embargo en los sitios en los cuales se incorporó las enmiendas orgánicas (T3 y T4) presentaron mayor salinidad. Tal como se puede observar en la Tabla 3, el testigo tuvo una $\mathrm{CE}$ de $1,1 \mathrm{dS} \mathrm{m}^{-1}$, vs el T3 $(\mathrm{CP})$ y T4 $(\mathrm{CCH})$ con 2,9 y 3,3 dS m${ }^{-1}$, respectivamente. Para el presente análisis es importante considerar que concentraciones salinas superiores a 2-2,5 dS m ${ }^{-1}$ pueden afectar el crecimiento y rendimiento del cultivo de tomate (Nuez et al., 2003; Porta et al., 2003; Maggio et al., 2007).

En F5 del ciclo 2015 no hubo diferencia significativa entre tratamientos (Tabla 3). No obstante al igual que el 2014, los T3 y T4 presentaron la mayor salinidad. Estos resultados coinciden con Barahona et al. (2015), quienes encontraron aumento de la salinidad al incorporar estiércol de gallina para biofumigar. En F6 el T4 (CCH) con mayor concentración (5,5 $\left.\mathrm{dS} \mathrm{m}^{-1}\right)$ se diferenció $(\mathrm{P}<0,05)$ del resto de los tratamientos. Por lo tanto la dosis implementadas en el ensayo, establecida en función del control de enfermedades y nematodos del suelo, pudo tener efectos negativos en las propiedades edáficas y resultar fitotóxicas (Oka, 2010). Las

Tabla 3. Valores de CE $\left(\mathrm{dS} \mathrm{m}^{-1}\right)$ para cada tratamiento y fecha de evaluación.

Table 3. EC values $\left(\mathrm{dS} \mathrm{m}^{-1}\right)$ for each treatment and season evaluated.

\begin{tabular}{lccccccc}
\hline & T1 & T2 & T3 & T4 & T5 & T6 & CV \\
\hline & & & \multicolumn{7}{c}{$\mathbf{2 0 1 4}$} & & \\
\hline F2 & $1,50 \mathrm{Aa}$ & $1,51 \mathrm{Aa}$ & $1,66 \mathrm{Aa}$ & $2,28 \mathrm{Aa}$ & $1,49 \mathrm{Aa}$ & $1,89 \mathrm{Aa}$ & 38,24 \\
F3 & $1,10 \mathrm{Aa}$ & $1,99 \mathrm{Aa}$ & $2,85 \mathrm{Aa}$ & $3,37 \mathrm{Aa}$ & $2,08 \mathrm{Aa}$ & $1,1 \mathrm{Aa}$ & 70,75 \\
CV & 32,98 & 117,17 & 50,17 & 43,24 & 66,94 & 28,94 & \\
\hline \multicolumn{7}{c}{$\mathbf{2 0 1 5}$} \\
\hline F4 & 1,43 & 1,1 & 2,35 & 0,96 & 1,67 & 1,76 & \\
F5 & $1,61 \mathrm{Aa}$ & $1,22 \mathrm{Aa}$ & $2,54 \mathrm{Aa}$ & $2,19 \mathrm{Aa}$ & $1,86 \mathrm{Aa}$ & $1,05 \mathrm{Aa}$ & 51,21 \\
F6 & $1,82 \mathrm{Aa}$ & $1,00 \mathrm{Aa}$ & $1,70 \mathrm{Aa}$ & $5,54 \mathrm{Ba}$ & $2,04 \mathrm{Aa}$ & $1,21 \mathrm{Aa}$ & 67,24 \\
CV & 69,53 & 10,14 & 45,2 & 40,1 & 75,45 & 30,3 & \\
\hline
\end{tabular}

Letras mayúsculas iguales entre tratamientos no difieren entre sí.

Letras iguales minúsculas entre fechas no difieren entre sí. 
concentraciones salinas de los sitios en los que se incorporó enmienda orgánica estuvieron en el mismo rango de valores a las reportadas por Cuellas (2017) (CE promedio $4 \mathrm{dS} \mathrm{m}^{-1}$ ) para suelos de la región.

En consecuencia la incorporación de enmiendas con elevada salinidad (Tabla 1), sumado al riego con agua de baja calidad (bicarbonatada sódica) y a las características edáficas (drenaje deficiente) provocaron salinización en los suelos con distinta intensidad. Similares resultados fueron encontrados por Alconada y Zembo (2000) y Alconada et al. (2011) al vincular la calidad del agua de riego, tipo de suelo, manejo, y rendimientos, con el nivel de salinización y alcalinización.

Sodicidad (RAS). Los valores de RAS para cada tratamiento y fecha de evaluación se presentan en la Tabla 4. Se observa que en ciclo 2014 la RAS se encontró entre 7,2 y 12,5 sin diferencia significativa entre tratamientos y fechas de muestreo, excepto para el testigo que tuvo un descenso significativo $(\mathrm{P}<0,05)$ desde F2 a F3.

En el 2015 la RAS tuvo en un rango más bajo que el ciclo anterior (entre 3,6 y 10,5) (Tabla 4). En F5 el T1 y el T4 con mayor y menor valor, respectivamente, se diferenciaron $(\mathrm{P}<0,05)$ del resto de los tratamientos (excepto el T4 del T6). La menor RAS del T4 se pudo deber a los valores elevados de $\mathrm{Ca}^{2+}$ que aportó la enmienda. Hacia F6 la RAS aumentan en todos los sitios, siendo significativo $(\mathrm{P}<0,05)$ para los $\mathrm{T} 4$, T5 y T6. Asimismo T5 y T6 con mayor valor se diferenciaron significativamente del resto de los tratamientos (Tabla 4).

Por lo tanto, no hubo en el presente estudio una tendencia clara en la evolución de la RAS para los diferentes tratamientos, lo que se puede atribuir a la elevada RAS del agua de riego, con alto contenido de bicarbonato y de $\mathrm{Na}^{+}$
(Alconada y Zembo, 2000), y a la variabilidad en la composición de las enmiendas orgánicas.

\section{Cationes solubles}

Calcio. La tendencia de las concentraciones de $\mathrm{Ca}^{2+}$ (meq L ${ }^{1}$ ) en el suelo para cada tratamiento en ambos ciclos de evaluación se presenta en la Fig. 1. Se observa que excepto por los T3 y T4 las concentraciones fueron similares entre sitios. El T4 $(\mathrm{CCH})$ presentó la mayor concentración respecto al resto de los tratamientos, siendo significativa $(\mathrm{P}<0,05)$ en el año 2015 (F5 y F6). Asimismo en este tratamiento se produjo un incremento en el tiempo (F2 8,8 a F6 $24 \mathrm{meq} \mathrm{L}^{-1}$ ). El T3 (CP) si bien tuvo un aumento hacia F5 llegando a valores de 13,5 meq $\mathrm{L}^{-1}$ en ningún caso fue significativo. Las mayores concentraciones observada en los T3 y T4 se debió a la composición que tienen las enmiendas (Tabla 1), que impactan directamente en la dinámica y contenido de los niveles de nutrientes del suelo (Di Gioia et al., 2017).

Cuellas y Alconada (2010) establecen para una adecuada nutrición valores de $\mathrm{Ca}^{2+}$ soluble entre 1 a 2 meq $\mathrm{L}^{-1}$; en ningún tratamiento las concentraciones fueron menores a este nivel, por lo tanto la nutrición en valores absolutos de $\mathrm{Ca}^{2+}$ fue adecuada. Esto posiblemente se debió a la riqueza natural de los suelos $\left(17,6\right.$ cmolc $\mathrm{kg}^{-1}$ en superficie) (Hurtado et al., 2006), a la acumulación de este elemento por fertilizaciones (orgánicas e inorgánicas) excesivas previas al estudio, situación muy común en la región (Alconada et al., 2011; Cuellas, 2017) y las características de las enmiendas incorporadas (Tabla 1).

Magnesio. La evolución de las concentraciones de $\mathrm{Mg}^{2+}$ para cada tratamiento en los dos ciclos de evaluación se presenta en la Fig. 2. Se observa que fue muy similar a la del $\mathrm{Ca}^{2+}$. Se destaca el T4 $(\mathrm{CCH})$ con los mayores valores, con diferencia significativa $(\mathrm{P}<0,05)$ para el

Tabla 4. Valores de RAS para cada tratamiento y fecha de evaluación. Table 4. RAS values for each treatment and season evaluated.

\begin{tabular}{|c|c|c|c|c|c|c|c|}
\hline & T1 & T2 & T3 & T4 & T5 & T6 & $\mathrm{CV}$ \\
\hline \multicolumn{8}{|c|}{2014} \\
\hline F2 & $12,46 \mathrm{aA}$ & $10,61 \mathrm{aA}$ & $11,53 \mathrm{aA}$ & $7,47 \mathrm{aA}$ & $9,99 \mathrm{aA}$ & $12,22 \mathrm{aA}$ & 20,46 \\
\hline F3 & $7,16 \mathrm{aA}$ & $8,75 \mathrm{aA}$ & $9,26 \mathrm{aA}$ & $7,47 \mathrm{aA}$ & $10,03 \mathrm{aA}$ & $8,2 \mathrm{aA}$ & 18,64 \\
\hline $\mathrm{CV}$ & 5,74 & 49,96 & 10,00 & 12,56 & 24,81 & 25,67 & \\
\hline \multicolumn{8}{|c|}{2015} \\
\hline F5 & $8,03 \mathrm{aC}$ & $5,93 \mathrm{aB}$ & $5,91 \mathrm{aB}$ & $3,62 \mathrm{aA}$ & $5,64 \mathrm{aB}$ & $4,93 \mathrm{aAB}$ & 19,55 \\
\hline F6 & $8,61 \mathrm{aAB}$ & $8,00 \mathrm{aA}$ & 7,96 aA & $7,55 \mathrm{bA}$ & $10,48 \mathrm{bB}$ & $8,99 \mathrm{bAB}$ & 12,02 \\
\hline $\mathrm{CV}$ & 34,62 & 13,28 & 23,2 & 26,04 & 18,08 & 21,44 & \\
\hline
\end{tabular}

Letras mayúsculas iguales entre tratamientos no difieren entre sí.

Letras iguales minúsculas entre fechas no difieren entre sí. 


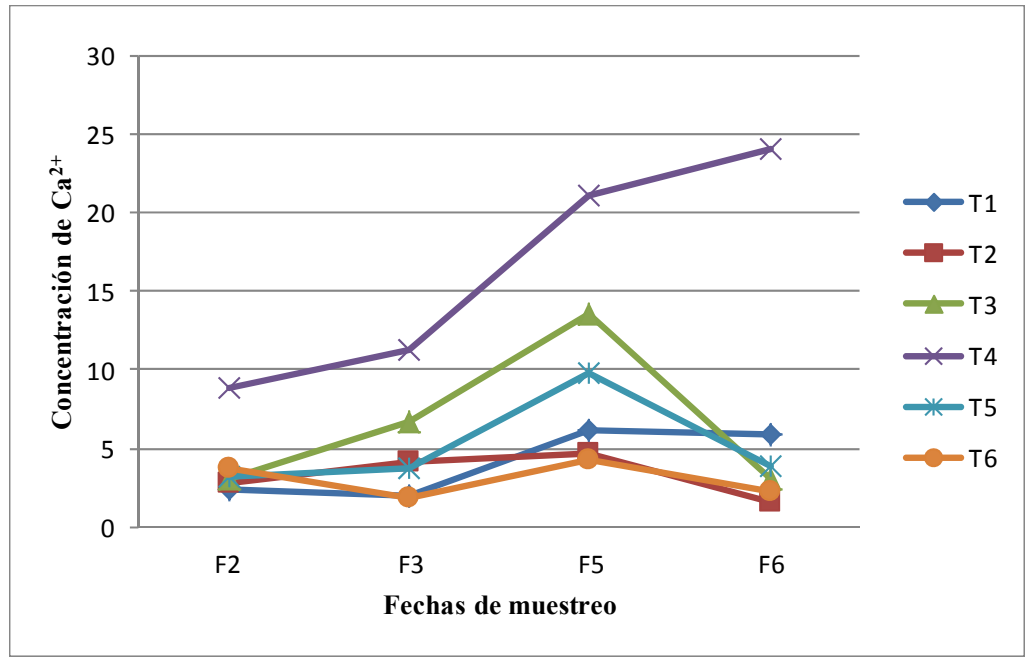

Fig. 1. Evolución de la concentración de $\mathrm{Ca}^{2+}\left(\right.$ meq $\left.\mathrm{L}^{-1}\right)$ en los dos ciclos de evaluación. Fig. 1. Evolution of $\mathrm{Ca}^{2+}$ concentration $\left(\mathrm{meq} \mathrm{L}^{-1}\right)$ in the two seasons evaluated.

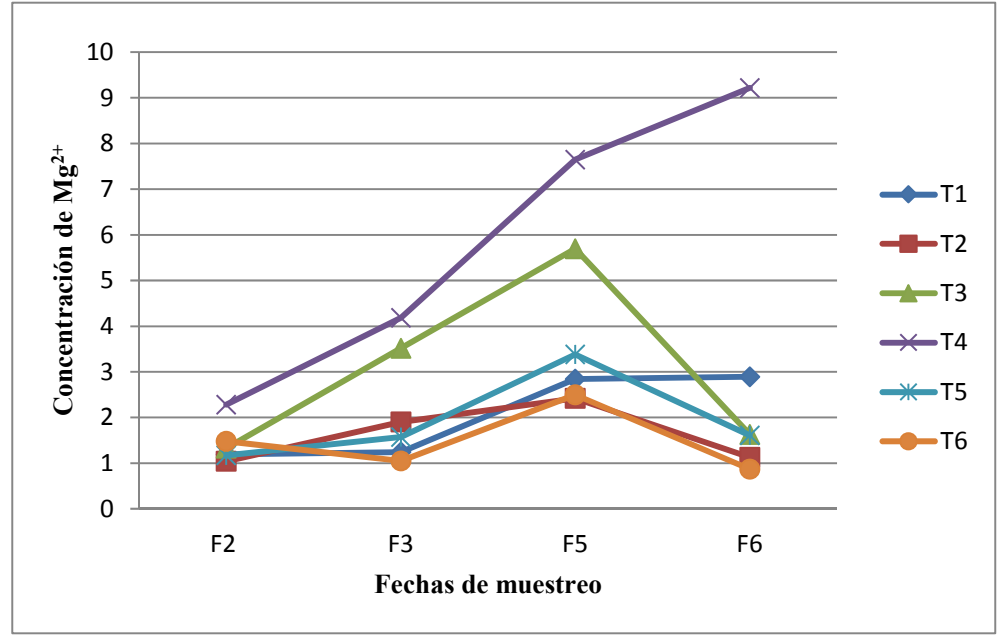

Fig. 2. Evolución de la concentración de $\mathrm{Mg}^{2+}\left(\mathrm{meq}^{-1}\right)$ en los dos ciclos de evaluación. Fig. 2. Evolution of $\mathrm{Mg}^{2+}$ concentration (meq $\mathrm{L}^{-1}$ ) in the two seasons evaluated.

2015, y con una tendencia de aumento hacia el final del ensayo llegando a concentraciones de 9,2 me L $^{-1}$. El T3 también tuvo un aumento hacia F5 (5,7 meq $\left.\mathrm{L}^{-1}\right)$ pero para ninguna fecha fue significativo. En los tratamientos en los cuales no se incorporó enmienda orgánica (T1, T2 y T6) las concentraciones estuvieron en un rango entre $0,9-2,9$ meq $\mathrm{L}^{-1}$. No obstante estos valores no condujeron a situaciones de deficiencia en el cultivo y se consideran suficientes (Cadahia, 2005). Cabe destacar que en condiciones de fertilización continua en suelos Hapludert típico de la región, las concentraciones de $\mathrm{Mg}^{2+}$ en la solución edáfica en general superan los $2 \mathrm{me} \mathrm{L}^{-1}$ hasta máximos de
13 meq L-1 (Alconada et al., 2006).

No solo los valores absolutos de $\mathrm{Ca}^{2+} \mathrm{y} \mathrm{Mg}^{2+}$ son importantes para asegurar la nutrición de los cultivos, sino que también a fin de evitar deficiencias nutritivas inducidas se requiere una adecuada relación entre ambos (Mengel and Kirkby, 2000). El $\mathrm{Mg}^{+2}$ se absorbe rápidamente por la raíz y compite por los sitios de absorción con el Ca ${ }^{+2}$ (Marschner, 1995). Se establece que si la relación $\mathrm{Ca} / \mathrm{Mg}$ es $<1$ habrá deficiencia de $\mathrm{Ca}^{+2}$, y si la misma es $>10$ la deficiencia será de $\mathrm{Mg}^{+2}$. En todos los sitios evaluados la relación promedio entre ambos cationes $(\mathrm{Ca} / \mathrm{Mg})$ estuvo entre 2 y 2,8. En consecuencia el rango fue óptimo 
y no se produjeron deficiencias inducidas entre ambos cationes.

Materia orgánica. La evolución de la MO (\%) para cada tratamiento y fechas de evaluación se presenta en la Fig. 3. Se observa al inicio una buena provisión de $\mathrm{MO}(4,5 \%)$, coincidente con lo reportado por Hurtado et al. (2006) para los suelos de la región. Una vez aplicados los tratamientos, el \% de MO tuvo un comportamiento similar en todos los sitios, con valores muy próximos entre sí sin diferenciarse significativamente. La falta de aumento significativo en los tratamientos con aplicación de enmienda orgánica (T3 y T4) se pudo deber a las características de la misma, debido a que la formación de humus se produce principalmente a partir de materiales de origen vegetal (Labrador Moreno, 1996). Las camas de pollo (estiércol mezclado con cascara de arroz) cuentan con carbono de difícil descomposición, con elevado contenido de sílice y cubierta cerosa en la cáscara, que impiden el humedecimiento y en consecuencia le confieren resistencia al ataque microbiano siendo muy difícil la formación de humus (del Pino et al., 2007).

Respecto al T5, si bien la biosolarización se realizó con repollo (material vegetal) la descomposición del mismo no produjo modificación del \% de $\mathrm{MO}$ en el suelo; esto posiblemente se deba a la dosis incorporada (80 $\mathrm{t} \mathrm{ha}^{-1}$ ), ya que la misma fue en base a lo recomendado para biofumigar (control de nematodos y enfermedades del suelo) (Bello et al., 2000) y no para provocar un efecto mejorador en el suelo. Por lo tanto, tal como lo manifiesta Barahona et al. (2015) la cantidad de enmienda a aplicar como biofumigante puede ser diferente en cada suelo, y se debe tener en consideración las características edáficas, siendo necesario realizar ensayos de diferentes dosis para determinar su efecto en el suelo y en la desinfección.

Rendimiento del cultivo y cantidad de frutos con BER. Los rendimientos del cultivo y la cantidad de frutos con BER para el 2014 y 2015 se presentan en la Tabla 5. Para ambos ciclos, tal como era de esperar el T6 (fumigante químico) tuvo los mayores rendimientos y esto se relaciona con la cantidad de nematodos y plantas muertas que se presentaron en este tratamiento (datos no mostrados). El resto de los tratamientos no presentaron diferencia significativa entre sí.

Respecto a la cantidad de frutos con BER no hubo diferencia significativa entre tratamientos. No obstante se observa en la Tabla 5 que en el ciclo 2014 todos los tratamientos presentaron un porcentaje menor que el testigo, y que por el contrario en el 2015 el porcentaje de frutos con BER fue mayor en los tratamientos. Se destaca en este año que los T3 y T4 (biosolarización con una enmienda orgánica) tuvieron el mayor porcentaje de BER, a pesar de tener en el suelo las mayores concentraciones de $\mathrm{Ca}^{2+}$, y además en el caso del T4 la salinidad llego a un valor de CE $5,5 \mathrm{dS} \mathrm{m}^{-1}$. Por lo tanto, tal como lo manifiestan diferentes autores (Ho and White, 2005; del Amor and Marcelis, 2006) la deficiencia de $\mathrm{Ca}^{2+}$ en los frutos en muchos casos no se debe a la falta de este catión sino a la interacción con otros cationes como el $\mathrm{Na}^{+}$(Rubio et al., 2009).

Por lo tanto, es muy importante continuar con investigaciones que permitan encontrar tipo

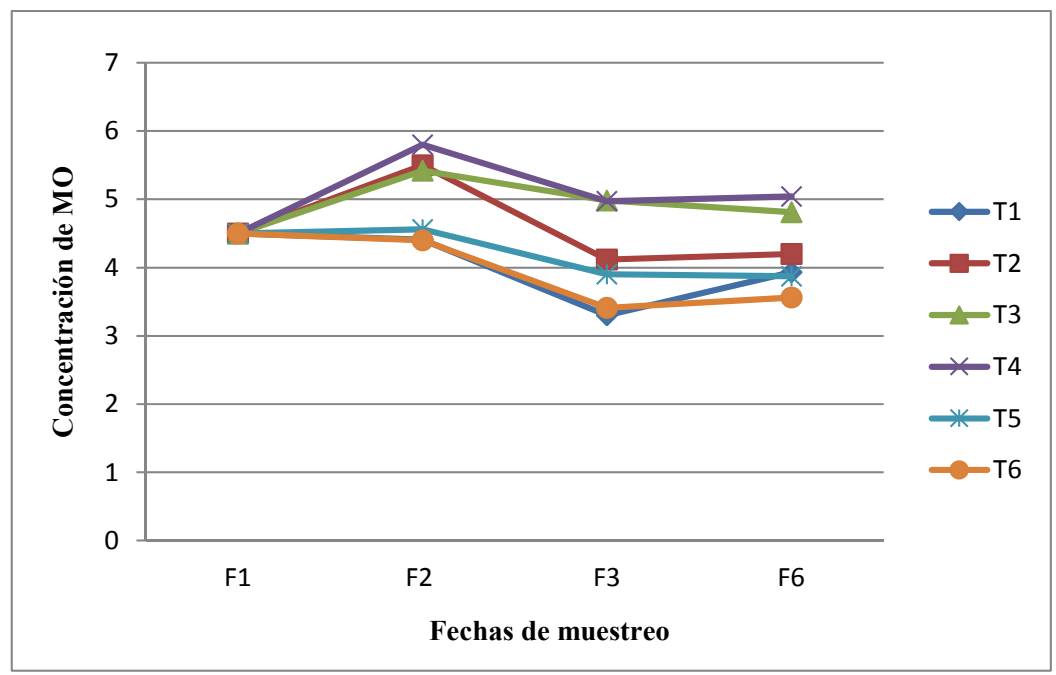

Fig. 3. Evolución de la concentración de MO (\%) en los dos ciclos de evaluación.

Fig. 3. Evolution of OM concentration (\%) in the two seasons evaluated. 
Tabla 5. Resultados de rendimiento $\left(\mathrm{kg} \mathrm{ha}^{-1}\right)$ y porcentaje (\%) de frutos con BER para cada tratamiento y ciclo de evaluación.

Table 5. Results of yield $\left(\mathrm{kg} \mathrm{ha}^{-1}\right)$ and percentage (\%) of fruits with BER for each treatment and season.

\begin{tabular}{lllll}
\hline \multicolumn{4}{c}{2014} & \multicolumn{2}{c}{$\mathbf{2 0 1 5}$} \\
\hline & R. $\left(\mathbf{k g ~ h a}^{-1}\right)$ & BER (\%) & R. $\left(\mathbf{k g ~ h a}^{-1}\right)$ & BER (\%) \\
\hline T1 & $29.682 \mathrm{~A}$ & 28,0 A & $32.250 \mathrm{~A}$ & $11,0 \mathrm{~A}$ \\
T2 & $39.405 \mathrm{AB}$ & $25,0 \mathrm{~A}$ & $35.400 \mathrm{~A}$ & $16,0 \mathrm{~A}$ \\
T3 & $36.114 \mathrm{~A}$ & $27,0 \mathrm{~A}$ & $33.450 \mathrm{~A}$ & $22,5 \mathrm{~A}$ \\
T4 & $33.195 \mathrm{~A}$ & $22,5 \mathrm{~A}$ & $32.850 \mathrm{~A}$ & $20,0 \mathrm{~A}$ \\
T5 & $37.380 \mathrm{~A}$ & $25,4 \mathrm{~A}$ & $36.433 \mathrm{~A}$ & $17,3 \mathrm{~A}$ \\
T6 & $49.537 \mathrm{~B}$ & $27,0 \mathrm{~A}$ & $51.109 \mathrm{~B}$ & $14,0 \mathrm{~A}$ \\
CV & 12,2 & 41,1 & 13,9 & 49,1 \\
\hline
\end{tabular}

R. Rendimiento; BER (blossom end rot); CV (coeficiente de variabilidad) Letras mayúsculas iguales entre tratamientos no difieren entre sí.

y dosis de enmiendas orgánicas que preserven los sistemas productivos de la región, y que promuevan su adecuado uso.

\section{CONCLUSIONES}

Las enmiendas orgánicas como la cama de pollo y de champiñón, incorporadas en el presente estudio para biofumigar el suelo, provocaron un aumento de la salinización que condujo en algunos casos, a pesar de presentar las mayores concentraciones de $\mathrm{Ca}^{2+}$ en el suelo, a aumentar la cantidad de frutos con blossom end rot. Asimismo en estos tratamientos no se encontró una mejora significativa en los niveles de materia orgánica del suelo.

La biosolarización con repollo a la dosis utilizada $\left(80\right.$ tha $\left.^{-1}\right)$ no produjo un efecto mejorador en las propiedades del suelo analizadas.

Los tratamientos con solarización y fumigación química no provocaron cambios significativos en las propiedades edáficas comportándose de manera similar al testigo.

Respecto a las variables de $\mathrm{pH}$ y RAS, para el pH se observó un descenso en los tratamientos con enmiendas, luego de transcurrido un tiempo se detecta una alcalinidad generalizada, que al igual que lo sucedió con la RAS, se mantuvieron en un mismo orden de valores entre los tratamientos, lo cual se asocia a la calidad del agua de riego y la calidad de las enmiendas utilizadas.

Asimismo no se observó una mejora en el rendimiento del cultivo en los tratamientos de biosolarización ensayados.

\section{RECONOCIMIENTOS}

Al Ing. Agr. Néstor Mezquiri, ex director de la Chacra Experimental de Gorina, y al personal de campo.
A los Ing. Agr. Guillermo Cap, Andrés Polack y M. Eugenia Strassera de la EEA INTA AMBA.

\section{LITERATURA CITADA}

Alconada, M. 2004. Desinfección del suelo con vapor. Efectos sobre la nutrición de los cultivos. 124 p. Proyecto Tierra Sana MP/ARG/00/033. INTA, Ciudad Autónoma de Buenos Aires, Argentina.

Alconada, M., P. Poncetta, M. Cuellas, S. Barragán, E. Inda, y A. Mitidieri. 2006. La fertirrigación en cultivo de tomate protegido (Lycopersicum esculentum): consecuencias ambientales, productivas y económicas. En XX Congreso Argentino de la Ciencia del Suelo, y I Reunión de Suelos de la región Andina. 20-22 de Septiembre. Asociación Argentina de Ciencia del Suelo (AACS), Salta, Argentina.

Alconada, M., M. Cuellas, P. Poncetta, S. Barragán, E. Inda, y A. Mitidieri. 2011. Fertirrigación en un cultivo de tomate protegido: I-Nutrición nitrogenada. Efectos en el suelo y en la producción. Revista Horticultura Argentina 30(72):5-13.

Alconada, M., y J. Zembo. 2000. Influencia cualitativa del riego con aguas subterráneas en suelos con producciones intensivas a campo y en invernáculo. En Actas 1er Joint World Congress on Groundwater. 31 de Julio-4 de Agosto. Northeastern Brazil Groundwater Project (PROASNE). Associação Brasileira de Aguas Subterrâneas (ABAS), Fortaleza, Brasil.

Altieri, M., and C. Nicholls. 2003. Soil fertility management and insect pests: harmonizing soil and plant health in agroecosystems. Soil \& Tillage Research 72:203-211. 
Auge, M., R. Hirata, y F. López 2004. Vulnerabilidad a la contaminación por nitratos del acuífero puelche en La Plata, Argentina. 187 p. Informes del Centro de Estudios de América Latina (CEAL). Disponible en http://tierra.rediris.es/hidrored/ebooks/miguel/Nitratos.pdf (Consulta 10 abril 2016).

Bello, A., J.A. López-Pérez, y L. Díaz-Veruliche. 2000. Biofumigación y solarización como alternativas al bromuro metilo. En Memorias del Simposium Internacional de la Fresa. 25 p. J.Z. Castellanos y F. Guerra (eds.) Gobierno del Estado de Michoacán, Zamora, México.

Bello, A., J.A. López-Pérez, M.A. Diez Rojo, J. López Cepero, y A.García-Álvarez. 2008. Principios ecológicos en la gestión de los agrosistemas. Arbor Vol. 184 (729):19-29.

Barahona, L.A., R. Samaniego, J. Guerra, G. Castillo, y J. Agurto. 2015. Utilización de la gallinaza como biofumigante de suelo en el cultivo de melón. Ciencia Agropecuaria 23:95-109.

Bonanomi, G., V. Antigmani, C. Pane, and F Scala. 2007. Suppression of soilborne fungal diseases with organic amendments. J. Plant Pathol. 89(3):311-324.

Cadahia, L.C. 2005. Fertirrigación: Cultivos hortícolas y ornamentales. 681 p. 3a. ed. Mundi Prensa, Madrid, España.

Cuellas, M. 2015. Control de la salinización del suelo mediante sistemas de drenes en producciones intensivas de cultivos bajo cubierta. 162 p. M. Sc. Tesis. Facultad de Agronomía, Universidad de Buenos Aires, Argentina.

Cuellas, M. 2017. Horticultura periurbana, análisis de la fertilidad de los suelos en invernaderos. Chilean J. Agric. Anim. Sci, ex Agro-Ciencia 33(2):163-173.

Cuellas, M., y M. Alconada. 2010. Diagnóstico de la nutrición de Ca-K en cultivos intensivos en extractos de suelo obtenidos en laboratorio y a campo. En XXII Congreso Argentino de la Ciencia del Suelo. 31 de mayo al 4 de junio, Rosario. Argentina. Asociación Argentina Ciencia de Suelo (AACS), Buenos Aires, Argentina.

del Amor, F.M., and L.F.M. Marcelis. 2006. Differential effect of transpiration and Ca supply on growth and $\mathrm{Ca}$ concentration of tomato plants. Scientia Horticulturae 111(1):17-23.

Del Pino, A., C. Repetto, C. Mori, y C. Perdoma. 2007. Patrones de descomposición de estiércoles en el suelo. Terra Latinoamericana 26:4352.

Di Gioia, F., M. Ozores-Hampton, X. Zhao, J. Thomas, P. Wilson, Z. Li, et al. 2017. Anaerobic soil disinfestation impact on soil nutrients dynamics and nitrous oxide emissions in fresh-market tomato. Agr. Ecosyst Environ. 240:194-205.
Dikinya, O., and N. Mufwanzala. 2010. Chiken manure-enchanced soil fertility and productivity: Effects of application rates. J. Soil Sci. Environ. Manag. 1(3):46-54.

Fernández, L., J. Pascuas, y A. Lacasa. 2014. Estudio de lixiviación de nitratos en suelos de invernaderos de pimiento biosolarizados. III Worshop en investigación agroalimentaria. 12-13 Mayo, Cartagena, Colombia. Disponible en http://repositorio.upct.es/bitstream/ handle/10317/4911/eln.pdf?sequence $=1$ (Consulta 20 marzo 2018).

Giuffré, L., M. Alconada, C. Pascale, and S. Ratto. 2004. Environmental impact of phosphorus over fertilization in tomato greenhouse production. Journal Applied Horticulture 6(1):58-61.

Grattan, S. R., and C. M. Grive. 1999. Salinity-mineral nutrient relations in horticultural crops. Scientia Horticulturae 78:127-157.

Ho, L.C., and P. White. 2005. A cellular hypothesis for the induction of blossom-end rot in tomato fruit. Ann. Bot. 95:571-581.

Hurtado, M., J. Giménez, y M. Cabral. 2006. Análisis ambiental del partido de La Plata: Aportes al ordenamiento territorial. $134 \mathrm{p}$. Consejo Federal de Inversiones, La Plata, Buenos Aires, Argentina.

InfoStat. 2004. Manual del usuario. Grupo InfoStat, FCA, Universidad Nacional de Córdoba. 318 p. Editorial Brujas, Córdoba, Argentina.

Labrador Moreno, J. 1996. La materia orgánica en los agroecosistemas. 174 p. Mundi Prensa, Madrid, España.

Maggio A., G. Raimondi, A. Martino, and S. De Pascale. 2007. Salt stress response in tomato beyond the salinity tolerance threshold. Environ. Exp. Bot. 59:276-282.

Marschner, H. 1995. Mineral nutrition of higher plants. 889 p. 2a. ed. Academic Press, San Diego, California, USA.

Mengel, K., and E.A. Kirkby. 2000. Principios de nutrición vegetal. 535 p. 4a. ed. Instituto Internacional de la Potasa, Basilea, Suiza.

Miranda, M. 2017. Riesgos ambientales al cultivo bajo cubierta en el cinturón hortícola del gran La Plata. En III Congreso Nacional de Ciencia y Tecnología Ambiental. Del 31 de Julio al 3 de Agosto. Santa Fe, Argentina. Sociedad Argentina de Ciencia y Tecnología Ambiental (SACyTA), Buenos Aires, Argentina.

Mitidieri, M., V. Brambilla, V. Saliva, E. Piris, M. Piris, R. Celie, et al. 2009. Efecto de distintas secuencias de tratamientos de biofumigación sobre parámetros fisicoquímicos y biológicos del suelo, el rendimiento y la salinidad de cultivos de tomate y lechuga bajo cubierta. Revista Horticultura Argentina 28(67): 5-7. 
Nuez, F., G. Ortega, y J. Costa. 2003. El cultivo de pimientos, chiles y ajíes. 607 p. Mundi Prensa, Madrid, España.

Oka, Y. 2010. Mechanisms of nematode suppression by organic soil amendments - A review. Appl. Soil Ecol. 44:101-105.

Page, A., A.H. Miller, and D.R. Keeney. 1982. Methods of soils analysis. Soil Sci. Soc. America, Madison, Wisconsin, USA.

Pérez, A., C. Céspedes, y P. Muñoz. 2008. Caracterización física-química y biológica de enmiendas orgánicas aplicadas en la producción de cultivos en República Dominicana. R. C. Suelo Nutr. Vegetal 8(4):10-29.

Ploeg, A.T., U.C. Riverside, and J.J. Stapleton. 2001. The effects of temperatura, time and amendment of soil with broccoli residues on the infestation of melos (Cucumis melo L.) by two root-knot nematode species. UC Plant Protection Quaterly. Disponible en www. uckac.edu/ppq (Accessed 5 December 2016).

Polack, L.A. 2013. Tecnología apropiada para la sustentabilidad de sistemas hortiflorícolas con énfasis en cultivos protegidos. (PNHFA 1106082). PTR 2013-2019. Instituto Nacional de Tecnología Agropecuaria (INTA), Buenos Aires, Argentina.

Poncetta, P., M. Alconada, y R. Lavado. 2006. Producción de tomate protegido en suelos decapitados con diferentes planes de fertirrigación. En XX Congreso Argentino de la Ciencia del Suelo y I Reunión de Suelos de la región Andina. 20-22 de Septiembre. Asociación Argentina de Ciencia del Suelo (AACS), Salta, Argentina.
Porta, J., M. López Acevedo, y C. Roquero. 2003. Edafología para la agricultura y el medio ambiente. 930 p. 3a. ed. Mundi Prensa, Madrid, España.

Rabuffetti, A., C. García, R. Docampo, S. Casanova, M. Moura, C. Smolark, et al. 2010. Evaluación agronómica y ambiental del estiércol de ave como fuente de nitrógeno en sistemas de producción intensiva. Seminario de actualización técnica. Manejo sustentable para la producción hortícola sustentable. 79 p. Serie Actividades de Difusión $\mathrm{N}^{\circ} 624$. Unidad de comunicación y transferencia de tecnología de INIA, Las Brujas. Canelones, Uruguay.

Rubio, J.S., F. García-Sánchez, F. Rubio, and V. Martínez. 2009. Yield, blossom-end rot incidence, and fruit quality in pepper plants under moderate salinity are affected by $\mathrm{K}^{+}$ and $\mathrm{Ca}^{2+}$ fertilization. Scientia Horticulturae 119:79-87.

Simmons, W., B. Higgins, S. Staley, L. Joh, B. Simmons, S. Singer, et al. 2016. The role of organic matter amendment level on soil heating, organic acid accumulation, and development of bacterial communities in solarized soil. Appl. Soil Ecol. 106:37-46.

Soil Survey Staff. 2006. Claves para la Taxonomía de Suelos. 331 p. USDA, Departamento de Agricultura, Washington, D.C, USA. 